\title{
Active Noise Compensation for Multichannel Magnetocardiography in an Unshielded Environment
}

W.A.M. Aarnink, P.J. van den Bosch, T.-M. Roelofs, M. Verbiesen, H.J. Holland, H.J.M. ter Brake and H. Rogalla, University of Twente, Applied Physics Department, Low Temperature Division, P.O. Box 217, 7500 AE Enschede, The Netherlands

\begin{abstract}
A multichannel high-T - -SQUID-based heart scanner for unshielded environments is under development. Outside a magnetically shielded room, sensitive SQUID measurements are possible using gradiometers. However, it is difficult to realize largebaseline gradiometers in high- $\mathbf{T}_{\mathrm{c}}$ materials. Therefore, we developed two active noise compensation techniques. In the Total Field Compensation technique, a Helmholtz type coil set is placed around the sensors. One magnetometer is used as a zero detector controlling the compensation current through the coil set. For Individual Flux Compensation, the reference signal is sent to the separate SQUIDs (or their flux transformer circuits) to compensate the local environmental noise fluxes. The latter technique was tested on low- $T_{c}$ rf-SQUID magnetometers, each sensor set to a field resolution typical for high-T $T_{c}$ SQUID magnetometers, i.e. $0.1 \mathrm{pT}_{\mathrm{RMS}} / \sqrt{H z}$. We were able to suppress the environmental disturbances to such an extent that magnetocardiograms could be recorded in an ordinary environment. In the paper the two suppression techniques are described and experimental results are presented.
\end{abstract}

\section{INTRODUCTION}

Up to now, superconducting quantum interference devices (SQUIDs) are the most sensitive magnetometers. Only with these devices biomagnetic signals (human heart, brain) can be detected. Low- $T_{c}$ SQUIDs have been widely applied for magnetocardiography (MCG) as well as magnetoencephalography (MEG) [1- 3].

Presently we aim at the development and realization of a high- $T_{c}$ SQUID based prototype heart scanner. The system should detect the biomagnetic signal of the (fetal) human heart in a magnetically unshielded environment (e.g. in a clinic or hospital). The system consists of high- $T_{c}$ SQUID magnetometers, a cooling subsystem and an environmental noise suppression subsystem.

The biomagnetic signal level of the fetal human heart is $1-10 \mathrm{pT}_{\mathrm{pp}}$ and of the adult human heart $10-100 \mathrm{pT}_{\mathrm{pp}}[4,5]$. If we want to perform magnetocardiography (MCG) in a frequency range of $0.1-40 \mathrm{~Hz}$, we need a magnetometer resolution of about $0.1 \mathrm{pT}_{\mathrm{RMS}} / \sqrt{\mathrm{Hz}}$. The heart signal, both adult and fetal, is very small compared to the magnetic noise

Manuscript received October 18, 1994

Supported by the Dutch National Research Program on High- $\mathrm{T}_{c}$ Superconductors and Philips GmbH Research Laboratories, Hamburg, FRG level in a typical laboratory or clinical environment (low frequency fluctuations in the order of a few $\mathrm{nT}$, about $10 \mathrm{nT}_{\mathrm{pp}}$ at $50 \mathrm{~Hz}$ ). Without precautions, the signal-to-noise ratio is far too low for biomagnetic measurements in an unshielded environment.

\section{PROBLEM DESCRIPTION}

For our purposes, a magnetically shielded room (MSR) is not useful. It is expensive and hardly transportable. Therefore, other ways to suppress environmental disturbances have to be developed.

One possibility to overcome problems with external magnetic fields would be the measurement of the magnetic field gradient instead of the magnetic field itself. Using superconducting wire one can build gradiometers measuring the gradient of the magnetic field and therefore, suppressing a uniform noise field from the environment. Employing a second order gradiometer, one is able to perform biomagnetic measurements in a magnetically unshielded environment [6].

High- $\mathrm{T}_{\mathrm{c}}$ SQUIDs have the advantage of a relatively high operating temperature. As a cooling agent, liquid nitrogen instead of liquid helium can be used. Liquid nitrogen has the advantages that it is cheap and relatively easy to handle. However, large-baseline gradiometers in high- $T_{c}$ materials cannot yet be realized in a simple manner. Therefore, active noise compensation techniques have to be incorporated in our high- $\mathrm{T}_{c}$ SQUID based heart scanner.

\section{ACTIVE NOISE COMPENSATION TECHNIQUES}

Active noise compensation techniques were applied before [7 - 11]. We tested two techniques by means of flux gate magnetometers (FGMs) and low- $T_{c}$ rf-SQUIDs. The aim was to investigate whether these techniques could be applied for high- $\mathrm{T}_{\mathrm{c}}$ SQUID magnetometers. In this paper the two techniques are described and noise measurements as well as a magnetocardiogram are presented and discussed.

\section{A. Requirements for noise compensation techniques}

Above we already saw that the environmental disturbances are much stronger than the magnetic signal of the human heart. Environmental disturbances have to be suppressed $40-60 \mathrm{~dB}$ for magnetocardiography (MCG) and $60-80 \mathrm{~dB}$ for fetal magnetocardiography (FMCG). 


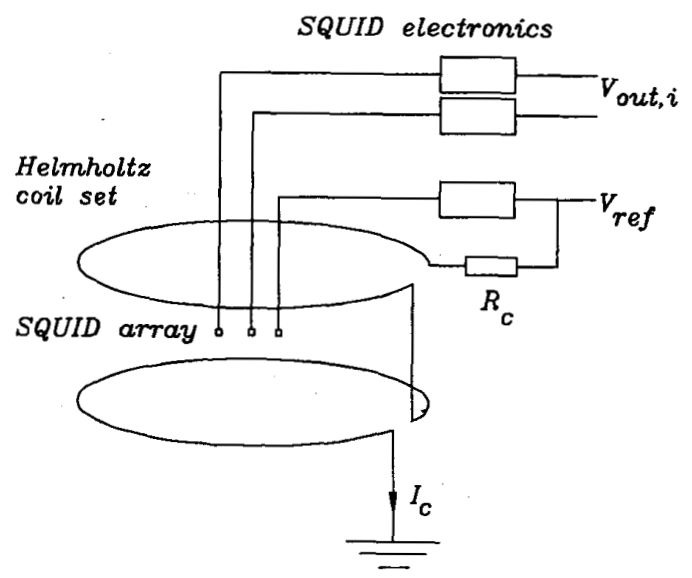

Fig. 1. Total field compensation (TFC).

\section{B. Total Field Compensation}

Total field compensation (TFC) can be applied for the improvement of a $\mu$-metal magnetically shielded room [7]. This technique may also be used to compensate disturbing environmental magnetic fields, see Fig. 1. Inside a large Helmholtz coil set an array of SQUID magnetometers is placed. One sensor is used as a zero detector controlling the compensation current through the coils. We tested this compensation technique using FGMs. Suppression factors of $20-30 \mathrm{~dB}$ were observed. One of the disadvantages is the difficulty to produce a uniform compensation field near the sensors. For a large sensor array this leads to large compensation coils, decreasing the transportability of the system. Therefore, TFC is less suited for our heart scanner.

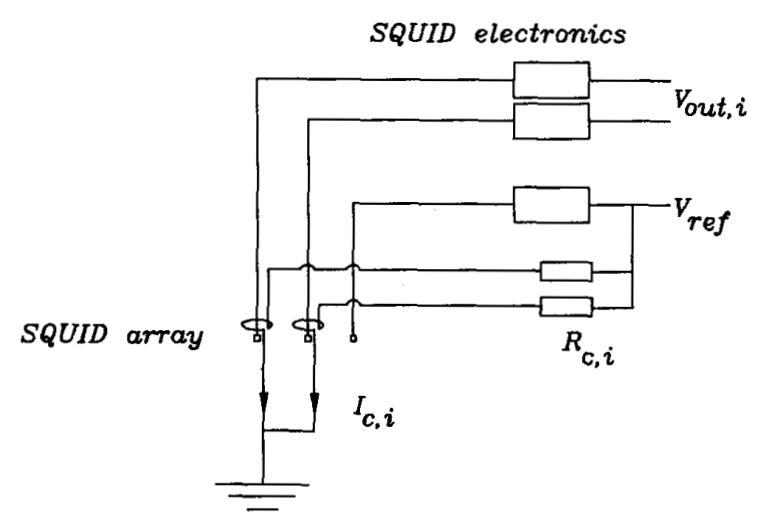

Fig. 2. Individual flux compensation (IFC).

\section{Individual Flux Compensation}

Instead of using a large Helmholtz set, one also can use separate small coils near the magnetometers to locally suppress environmental disturbances, see Fig. 2. The output of one of the channels is used as a noise reference and sent to the separate SQUIDs (or their flux transformer circuits) to compensate the local environmental noise fluxes. Therefore, this technique is called individual flux compensation (IFC). Compensation coils and magnetometers can be integrated e.g. in a small tube, enabling the development of a modular system. In such a system, channels can easily be added and serviced, if necessary. Also the dimensions of the system can be smaller, favouring a transportable set-up. This technique was tested with low- $T_{c}$ rf-SQUIDs, as is discussed in the following section.

\section{APPLICATION OF INDIVIDUAL FLUX COMPENSATION}

\section{A. Experimental set-up}

In the future, we want to apply IFC on high- $\mathrm{T}_{\mathrm{c}}$ SQUIDs. We developed our magnetometers, which are based on low- $T_{c}$ SQUIDs, in such a way that their intrinsic noise levels are comparable to high- $T_{c}$ SQUID magnetometers. Our results then are indicative of what can be achieved with the combination of high- $\mathrm{T}_{\mathrm{c}}$ SQUIDs and IFC.

The IFC technique was tested with two low- $\mathrm{T}_{\mathrm{c}}$ rf-SQUIDs [12] each connected to a magnetometer-type pick-up coil, see Fig. 3. The distance or baseline $b$ between the two pick-up coils was about $10 \mathrm{~cm}$. The transfer of each flux transformer was designed in such a way that the field resolution of the two channels equals the typical value of high- $T_{c}$ dc-SQUID magnetometers of $0.1 \mathrm{pT}_{\mathrm{RMS}} / \sqrt{\mathrm{Hz}}$ at $77 \mathrm{~K}[13,14]$. However, one should note that much lower values can be reached when applying low- $T_{c}$ rf-SQUIDs (e.g. see [15]). For the magneticfield-to-voltage transfer function $\Delta V / \Delta B$ of the magnetometers we may write:

$$
\frac{\Delta V}{\Delta B}=\frac{\Delta V}{\Delta \Phi_{\mathrm{SQ}}} \frac{\Delta \Phi_{\mathrm{SQ}}}{\Delta \Phi_{\mathrm{P}}} \frac{\Delta \Phi_{\mathrm{P}}}{\Delta B},
$$

where $\Phi_{\mathrm{SQ}}$ is the flux in the SQUID and $\Phi_{\mathrm{P}}$ the flux in the pick-up coil. For the SQUIDs we used, the flux-to-voltage transfer function $\Delta V / \Delta \Phi_{\mathrm{SQ}}$ equals $20 \mathrm{mV} / \phi_{0}[12]$, with $\phi_{0}$ the flux quantum. The flux transformer consisted of 3 pick-up windings with a radius of $7 \mathrm{~mm}$, inductively coupled to the SQUID via a mutual inductance $M$. In our case $M$ equals $20 \mathrm{nH}$, whereas the total flux transformer inductance $\mathrm{L}_{\mathrm{f}}$ equals $2.4 \mu \mathrm{H}$. Therefore: $\Delta \Phi_{\mathrm{SQ}} / \Delta \Phi_{\mathrm{P}}=\mathrm{M} / \mathrm{L}_{\mathrm{f}}=8.3 \cdot 10^{-3}$. The transfer function $\Delta \Phi_{\mathrm{P}} / \Delta \mathrm{B}$ equals $A_{P}$, the total area of the 3 pick-up windings (in our case, $A_{P}=4.62 \mathrm{~cm}^{2}$ ). Therefore, for the magnetic-field-to-voltage transfer function we find: $\Delta \mathrm{V} / \Delta \mathrm{B}=3.9 \cdot 10^{7} \mathrm{~V} / \mathrm{T}$. Above we used theoretical values to calculate $\triangle \mathrm{V} / \Delta \mathrm{B}$, in practice a relative error of about $\pm 20 \%$ may well occur in $\Delta V / \Delta B$, due to errors in $\Delta V / \Delta \Phi_{\mathrm{SQ}}$ and $\mathrm{M}$. 


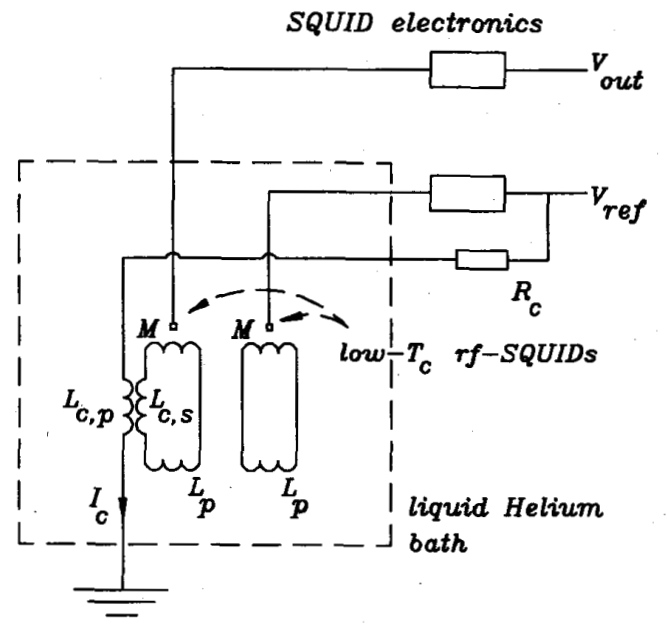

Fig. 3. Experimental set-up for testing IFC on low- $T_{c}$ rfSQUIDs.

In IFC, the signal of the reference channel is inductively fed into the flux transformer circuit of the measuring channel via a compensation resistance $R_{C}$, see Fig. 3. The mutual inductance $\mathrm{M}_{\mathrm{C}}$ that was used for this purpose consisted of a horse shoe shaped toroid (radius $4 \mathrm{~mm}$ ) with a primary and a secondary coil of 100 and 20 windings, respectively. Each winding had a radius of $1 \mathrm{~mm}$. The inductance $\mathrm{L}_{\mathrm{C}, \mathrm{P}}$ of the primary coil equaled $2 \cdot 10^{-6} \mathrm{H}$, the inductance $L_{C, S}$ of the secondary coil was $8 \cdot 10^{-8} \mathrm{H}$ and the mutual inductance $\mathrm{M}_{\mathrm{C}}$ was about $4 \cdot 10^{-7} \mathrm{H}$ (with the coupling constant $\mathrm{k}$ near 1 ). For the compensation current $I_{C}$ used for flux compensation, we may write, neglecting the impedance $\mathrm{L}_{\mathrm{C}, \mathrm{S}}$ of the primary coil:

$I_{\mathrm{C}}=\frac{\Delta V}{\Delta B} \frac{B_{\mathrm{ENV}}}{R_{\mathrm{C}}}$,

where $\mathrm{B}_{\mathrm{ENV}}$ is the environmental magnetic noise field. For optimal compensation (1st order gradiometer) the flux $\Phi_{C}$ should equal the flux $\Phi_{\mathrm{P}}=\mathrm{A}_{\mathrm{P}} B_{\mathrm{ENV}}$ in the pick-up coil of the signal line and we find:

$\Phi_{\mathrm{C}}=M_{\mathrm{C}} I_{\mathrm{C}}=\frac{\Delta V}{\Delta B} \frac{M_{C}}{R_{\mathrm{C}}} B_{\mathrm{ENV}}=A_{\mathrm{P}} B_{\mathrm{ENV}}$,

yielding a value of $33 \mathrm{k} \Omega$ for $R_{C}$. Again we used theoretical values to calculate $R_{C}$. Above we saw that in $\Delta V / \Delta B$ alone a relative error of $\pm 20 \%$ may occur. Therefore, relative errors in $R_{C}$ of about $\pm 30 \%$ may be expected, also due to an error in $\mathrm{M}_{\mathrm{C}}$.

\section{B. Noise considerations}

For the SQUIDs we used, the intrinsic SQUID flux noise level $\left(\mathrm{S}_{\Phi, S Q}\right)^{1 / 2}$ equaled $2 \cdot 10^{-4} \phi_{0} / \sqrt{\mathrm{Hz}}[12]$. For the equivalent flux noise $\left(\mathrm{S}_{\Phi, P}\right)^{1 / 2}$ in the pick-up coil we then may write:

$$
\left(S_{\Phi, \mathrm{P}}\right)^{1 / 2}=\frac{L_{\mathrm{f}}}{M}\left(S_{\Phi, \mathrm{SQ}}\right)^{1 / 2},
$$

and we find: $\left(\mathrm{S}_{\Phi, \mathrm{P}}\right)^{1 / 2}=2.4 \cdot 10^{-2} \phi_{0} / \sqrt{\mathrm{Hz}}$. This corresponds to a field noise level $\left(S_{B}\right)^{1 / 2}=\left(S_{\Phi, P}\right)^{1 / 2} / A_{P}$ of $0.1 \mathrm{pT}_{R M S} / \sqrt{H z}$ for each magnetometer channel.

In IFC, the intrinsic noise of the reference channel is fed into the measuring channels. For the corresponding flux noise level $\left(\mathbf{S}_{\Phi, \mathrm{C}}\right)^{1 / 2}$ of the compensation flux coupled into the flux transformer we find:

$\left(S_{\Phi, \mathrm{C}}\right)^{1 / 2}=\frac{\Delta V}{\Delta B} \frac{M_{\mathrm{C}}\left(S_{\mathrm{B}}\right)^{1 / 2}}{R_{\mathrm{C}}}$

This compensation flux noise adds to the intrinsic noise of the measuring channel. Since these are not correlated, we find for the theoretical flux noise level $\left(S_{\Phi, \text { IFC }}\right)^{1 / 2}$ in the measuring channel:

$\left(S_{\Phi, \mathrm{FC}}\right)^{1 / 2}=\left(S_{\Phi, \mathrm{P}}+S_{\Phi, \mathrm{C}}\right)^{1 / 2}$

and in our case this equals $3.4 \cdot 10^{-2} \phi_{0} / \sqrt{\mathrm{Hz}}$, corresponding to a field noise level $\left(\mathrm{S}_{\mathrm{B}, \mathrm{IFC}}\right)^{1 / 2}$ of $0.15 \mathrm{pT}_{\mathrm{RMS}} / \mathrm{JHz}_{\mathrm{Hz}}$

\section{Optimization of compensation settings}

For optimization and testing purposes, we put our set-up in a large Helmhoitz coil set-up (coil diameter $1.8 \mathrm{~m}$ ). We subjected our SQUID magnetometer system to a uniform harmonic magnetic field with a frequency of about $5 \mathrm{~Hz}$. The compensation resistance $R_{C}$ was tuned so that the amplitude of the resulting field at this frequency after compensation was minimal. With $R_{C}=43 \mathrm{k} \Omega$ we found suppression factors for the $5 \mathrm{~Hz}$ magnetic field of about $55 \mathrm{~dB}$. After tuning, the Helmholtz coil set was disconnected. Noise measurements have been performed with and without IFC, see Fig. 4. In a range of $0.5-20 \mathrm{~Hz}$, suppression factors for the environmental disturbing field of about $40 \mathrm{~dB}$ have been found. At higher frequencies suppression factors of $20-30$ $\mathrm{dB}$ have been measured. We see that above $3 \mathrm{~Hz}$ the noise

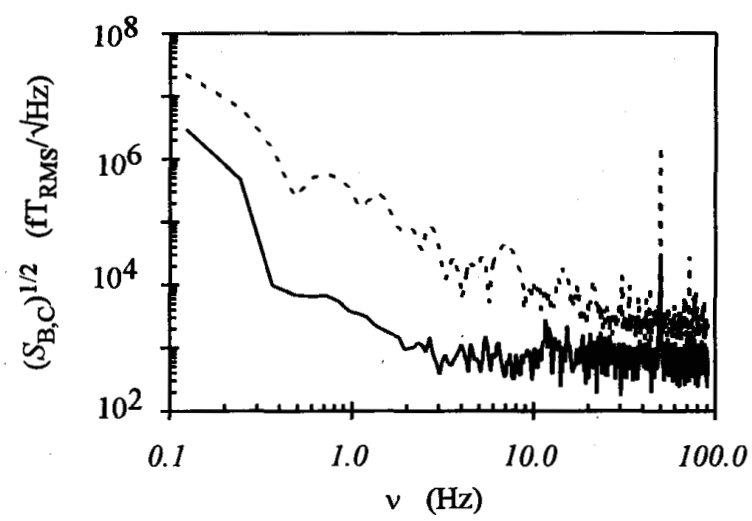

Fig. 4. Noise measurements with (solid line) and without (dashed line) IFC. 
level is around $0.5 \mathrm{pT}_{\mathrm{RMS}} / \sqrt{\mathrm{Hz}}$, a factor of 3 above the expected intrinsic noise level $\left(\mathrm{S}_{\mathrm{B}, \mathrm{IFC}}\right)^{1 / 2}=0.15 \mathrm{pT}_{\mathrm{RMS}} / \sqrt{\mathrm{Hz}}$ of the system. We speculate that gradients in the noise field combined with the fact that the two pick-up coils are not exactly coplanar, are the cause for this unexpected increase.

\section{Magnetocardiography in an unshielded environment}

From Fig. 4, we see that by applying IFC we can reach noise levels of about $0.5 \mathrm{pT} \mathrm{T}_{\mathrm{RMS}} / \sqrt{\mathrm{Hz}}$. This noise level is low enough to measure the magnetic heart signal of an adult. In Fig. 5, a magnetic heart signal as we recorded it, is shown. An analog 2nd order low pass filter with a cut-off frequency of $10 \mathrm{~Hz}$ was applied, followed by a digital filter removing the remaining peaks at 50 and $100 \mathrm{~Hz}$.

\section{DISCUSSION AND CONCLUSIONS}

We successfully applied IFC in combination with low- $T_{c}$ rf-SQUIDs for MCG in an unshielded environment. However, the noise level of our sensor is about a factor of 3 above the expected value. We expect to improve the field sensitivity by applying advanced gradiometric configurations (implemented in software) and by arranging the magnetometers in a perfectly coplanar way.

An attractive application of a heart scanner would be fetal magnetocardiography (FMCG). Application of electrocardiography is limited for the study of the fetal heart signal [16]. The magnetic signal level of the fetal heart is about a factor of 10 below that of an adult heart. We showed that with a resolution of $0.5 \mathrm{pT}_{\mathrm{RMS}} / \sqrt{\mathrm{Hz}}$ we can perform MCG. For FMCG we would need a system with a resolution of about 50 $\mathrm{fT}_{\mathrm{RMS}} / \sqrt{\mathrm{Hz}}$ to obtain a similar signal-to-noise ratio as in our present MCG. Therefore, also the improvement of the field sensitivity of high- $\mathrm{T}_{\mathrm{c}}$ SQUID magnetometers needs attention, if one wants to apply these sensors for FMCG.

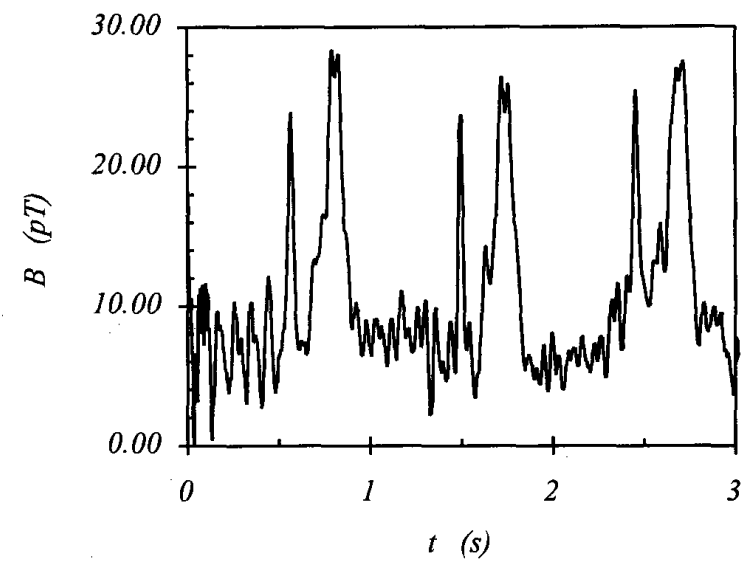

Fig. 5. Typical magnetocardiogram, taken in a magnetically unshielded environment using individual flux compensation.
For large sensor arrays in which IFC is applied, it will be of great importance that the sensors are coplanar. We did not yet investigate this in detail. In the future, the effect of deviations in this respect will be investigated on a multichannel system.

Our set-up effectively resembles a first order gradiometer. Higher order gradients of the disturbing environmental field are not removed. Therefore, nearby noise sources, producing higher order gradients, will be less effectively suppressed. This is especially the case when large sensor arrays with large baselines are used. Future studies also should concentrate on this subject.

We used a large Helmholtz coil set to tune our IFC. In a follow-up system correlation techniques for tuning the different channels will be applied.

\section{REFERENCES}

[1] S.J. Williamson, G.-L. Romani, L. Kaufman, and I. Modena, Eds., Biomagnetism an Interdisciplinary Approach, New York: Plenum Press, 1983.

[2] S.J. Williamson, M. Hoke, G. Stroink, and M. Kotani, Eds., Advances in Biomagnetism, New York: Plenum Press, 1989.

[3] M. Hoke, S.N. Erné, Y.C. Okada, and G.L. Romani, Eds., Biomagnetism Clinical Aspects, Amsterdam: Elsevier Science Publishers B.V., 1992.

[4] T. Katile and P. Karp, "Magnetocardiography: morphology and multipole presentations", in [1], pp. 237-263.

[5] V. Kariniemi and T. Katile, "Perinatal magnetocardiography", in [1], pp. 299-310.

[6] R.R. Fenici, G. Melillo, and M. Masselli, "Clinical magnetocardiography 10 years experience at the Catholic university", Int. J. of Cardiac Imaging, vol. 7, pp. 151-167, 1991.

[7] H.J.M. ter Brake, R. Huonker, and H. Rogalla, "New results in active noise compensation for magnetically shielded rooms", Meas. Sci. Technol., vol. 4, pp. 1370-1375, 1993.

[8] K. Fujioka, K. Matsumoto, Y. Yamagishi, T. Noda, and Y. Kuraoka, "Active shield with SQUID for biomagnetic measurement", in Superconducting Devices and Their Applications, H. Koch and H. Lübbig, Eds., Berlin: SpringerVerlag, 1992, pp. 533-536.

[9] H. Seifert, A. Wirth, and S. Schneider, "Active magnetic shielding support for biomagnetic instruments", in Superconducting Devices and Their Applications, $\mathrm{H}$. Koch and $\mathrm{H}$. Lübbig, Eds., Berlin: Springer-Verlag, 1992, pp. 537-541.

[10] K. Yokosawa and S. Kuriki, "Design and fabrication of directfeedback gradiometer", to be published in the Proc. of the 9th Int. Conf. on Biomagnetism, Vienna, 1993.

[11] R.H. Koch, J.R. Rozen, J.Z. Sun, and W.J. Gallagher, "Three SQUID gradiometer", Appl. Phys. Lett., vol. 63, pp. 403-405, 1993.

[12] Biomagnetic Technologies Inc., PO Box 210079, San Diego, CA 92121 , USA. System $330 \mathrm{x}$.

[13] J.W.M. Hilgenkamp, G.C.S. Brons, S. Hoogeveen, J. Flokstra, and H. Rogalla, "Monolithic flux transformer coupled high $\mathrm{T}_{c} \mathrm{dc}$ SQUID magnetometers", to be published in IEEE Trans. on Appl. Superc., 1994.

[14] D. Koelle, A.M. Micklich, F. Ludwig, E. Dantsker, D.T. Nemeth, and J. Clarke, "dc SQUID magnetometers from single layers of $\mathrm{YBa}_{2} \mathrm{Cu}_{3} \mathrm{O}_{7-\mathrm{x}}$ ", Appl. Phys. Lett, vol. 63, pp. 2271-2273, 1993.

[15] H.J.M. ter Brake,.P.J. van den Bosch, and H.J. Holland, "Magnetic noise of small Stirling coolers", in Advances in Cryogenic Engineering, vol. 39B, P. Kittel, Ed., New York: Plenum Press, 1994, pp. 1287-1295.

[16] Z. Dunajski and M. Peters, "Volume current effects on the fetal magnetocardiogram, in [3], pp. 565-569. 